\title{
Transformaciones socioculturales generadas por la convivencia con niños, adolescentes y jóvenes con Trastorno de Espectro Autista
}

\author{
DOI: http://dx.doi.org/10.5377/koot.v0i10.6701 \\ URI: http://hdl.handle.net/11298/787
}

Rodrigo Antonio Colorado

Antropólogo e investigador independiente

www.rodrigocolorado.com

\section{Resumen}

El presente trabajo es una investigación cuanti-cualitativa sobre la educación inclusiva en los centros educativos de El Salvador. Las administraciones de los presidentes Mauricio Funes 2009-2014 y Salvador Sánchez 2014-2019, han llevado consigo una serie de talleres de sensibilización en el Ministerio de Educación de El Salvador de aquí en adelante (MINED) sobre la inclusión educativa de estudiantes con autismo, así como también asociaciones como Angelitos, Asociación de Autismo de El Salvador de aquí en adelante (ASA), Music Time e instituciones que forman parte del Sistema Salud como el Instituto Salvadoreño de Rehabilitación Integral de aquí en adelante (ISRI) y el Hospital Bloom. Sin embargo, no existe un programa interinstitucional que permita llevar un control de los procesos educativos de los estudiantes con autismo. A pesar de las limitaciones institucionales, los estudiantes con el Trastorno de Espectro Autista de aquí en adelante (TEA), padres de familia, especialistas de la salud, instituciones gubernamentales y las asociaciones han sabido construir una cultura de Educación Inclusiva, la cual debe de ser fortalecida por un programa gubernamental en El Salvador.

Palabras Clave: Integración escolar; Segregación en la enseñanza; Trastorno de espectro autista; Autismo; Trastornos del desarrollo; Endoculturación.

\begin{abstract}
This is a qualitatite-quantitative research on inclusive education in the schools of El Salvador. The administration of presidents Mauricio Funes (2009-2014) and Salvador Sánchez (2014-2019), have included a series of awareness workshops at the Ministerio de Educación de El Salvador (Ministry of Education of El Salvador), hereon referred to as (MINED). Said workshops have approached
\end{abstract}


inclusive education for students with autism and have included associations such as Angelitos, the El Salvador Autism Association (ASA, given its Spanish acronym), Music Time as well as those institutions that are part of the Health System such as the Salvadorean Institute of Integral Rehabilitation (ISRI, given its Spanish acronym) and Hospital Bloom. However, there is no interinstitutional program to control the educational processes of students with Autism. In spite of the institutional limitations, those students within the Autism Spectrum Disorder (ASD), their parents, health professionals, governmental institutions and associations, have been able to build a culture of Inclusive Education, which must be strengthened by a state program in El Salvador.

Keywords: School integration; Segregation in teaching; Autistic spectrum disorder; Autism; Developmental disorders; Endoculturation.

\section{Introducción}

Este artículo de investigación antropológica estudia a los Centros Educativos, específicamente los entornos educativos y socioculturales en que se realiza el estudiante con autismo, partiendo de una arista holística que permite comprender en su totalidad la complejidad del proceso de aprendizaje del estudiante.

Los estudiantes con el TEA en El Salvador y el mundo tienen problemas sociales de discriminación al ser integrados e incluidos a la escuela regular, sin embargo, la labor de sensibilización de los padres de familia, asociaciones e instituciones gubernamentales ha permitido que docentes y directores de Centros Educativos logren recibir al estudiante, muy a pesar de la falta de formación académica en el tema.

En El Salvador, el discurso político de la Educación Inclusiva, rompe las barreras de la escuela tradicional, que por siglos ha negado las necesidades de los estudiantes, la escuela inclusiva es un proyecto naciente que se desarrolla con los programas educativos. En nuestro país a través del MINED y lo lleva a cabo con los siguientes programas:

1. Programa Presidencial de Dotación de Paquetes Escolares (MINED, 2015).

2. Sub-Programa Vaso de Leche (Ibídem).

3. Programa de Alimentación y Salud Escolar (Ibídem).

4. Programa presidencial un niño, una niña, una computadora (Ibídem). En este artículo de divulgación solo se presenta la evaluación de este programa social. 
Una Educación Inclusiva, se construye a partir de los derechos culturales y educativos que el estudiante tiene dentro de los Centros Educativos, así se garantiza la profundización de conocimientos adquiridos. El sinónimo de una Educación Inclusiva es la actualización de los métodos de enseñanza, para garantizar la satisfacción de las necesidades que el estudiantado presenta en el aula, en El Salvador es una tarea cotidiana de los docentes.

\section{Metodología}

El enfoque teórico de la actual investigación está forjado desde una metodología mixta cuanti-cualitativo, se caracteriza porque está fusionada con los enfoques de la investigación cuantitativa, que analiza las cantidades en número los resultados indagados y el enfoque cualitativo analiza la percepción que el investigador tiene sobre los entornos socioculturales, es decir, que incluye las características de cada uno de ellos (eumed.net, 2012). En el desarrollo de la investigación se utilizaron encuestas, guía de observación, historias de vida, entrevistas dirigidas y entrevistas semiestructuradas, estas fueron realizadas a 74 Docentes de Centros Educativos de los Departamentos de: San Salvador, Ahuachapán, Chalatenango, Sonsonate, La Libertad y San Vicente, la investigación no es cuantificable, es decir, es un pequeño grupo de encuestados seleccionados como muestra.

Por otro lado, el método etnográfico es el que se ha utilizado, desde el enfoque cualitativo se considera que indaga la vida de un grupo específico a investigar en este caso: Los padres de familia, estudiantes con TEA, pares, docentes, profesionales de la salud, doctores especialistas, organizaciones gubernamentales, organizaciones no gubernamentales, Centros Educativos y todos los involucrados en la socialidad del estudiante con el TEA (Rojas, 2003). Se aborda el problema desde la Antropología de la Educación, la cual aporta conocimientos sistemáticos porque lleva a cabo investigaciones sobre los modos, valores y "estilos de vida" de quien se educa, sobre el proceso y sobre los contextos e instituciones donde se lleva a cabo la educación, desde una perspectiva antropológica y con unos métodos concretos (Geta, 2011).

\section{Teoría}

A continuación, se presentan los conceptos que han sido aplicados a la presente investigación, el discurso teórico de la Educación Inclusiva de la UNESCO, Manual Diagnóstico y Estadístico de los Trastornos Mentales y demás Ciencias Sociales. 


\section{El Trastorno de Espectro Autista}

La conceptualización más integral del autismo es el Trastorno de Espectro Autista (TEA). En Estados Unidos el Instituto Nacional de Salud Infantil y Desarrollo Humanos considera que es una condición neurológica y de desarrollo que comienza en la niñez y dura toda la vida. Afecta cómo una persona se comporta, interactúa con otros, se comunica y aprende.

Este trastorno incluye lo que se conocía como síndrome de Asperger y el trastorno generalizado del desarrollo no especificado (Medline Plus, 2016). En la vida cotidiana las personas con el TEA, son representadas por el conocimiento popular de las personas que las rodean es decir los grupos sociales, a este tipo de conocimiento la llamaremos "Las representaciones sociales", esta es una forma de conocimiento a través de la cual quien conoce se coloca dentro de lo que conoce (Mora, 2002). En este tema de investigación en el análisis cualitativo se ha optado por abordar las representaciones sociales que tienen los padres de familia, los especialistas de la salud, docentes y estudiantes pares sobre el estudiante con autismo, la visión que tiene el estudiante TEA sobre su entorno, así como la evaluación de los programas sociales del Gobierno de El Salvador con los estudiantes con TEA y los estudiantes "regulares", son los que no están diagnosticados con ninguna Necesidad Educativa Especial.

\section{Proceso de aprendizaje del estudiante con autismo.}

Todos los seres humanos cuando nacemos, comenzamos a percibir el mundo a través de nuestros sentidos, toda experiencia que tenemos en la vida la llamaremos endoculturación, es una experiencia de aprendizaje parcialmente consciente y parcialmente inconsciente a través de la cual la generación adulta, educa a los niños para que estos adopten las tradiciones de las generaciones de sus antepasados y con ello sus modos de pensar (Harris, 2004).

Del proceso de endoculturación no escapan las personas con autismo, ya que aprenden desde la niñez todo el conocimiento que se les ha enseñado a través de pictogramas, consignas, etc., dicho conocimiento le posibilita la comunicación con la sociedad.

\subsection{Aprendizaje experiencial.}

Como hemos expresado sobre el proceso de aprendizaje del ser humano, es un proceso experiencial y por tanto este solo se puede desarrollar en la sociedad. Al proceso de aprendizaje se le hermana, el concepto socialidad (Och y Solomon, 2010). 


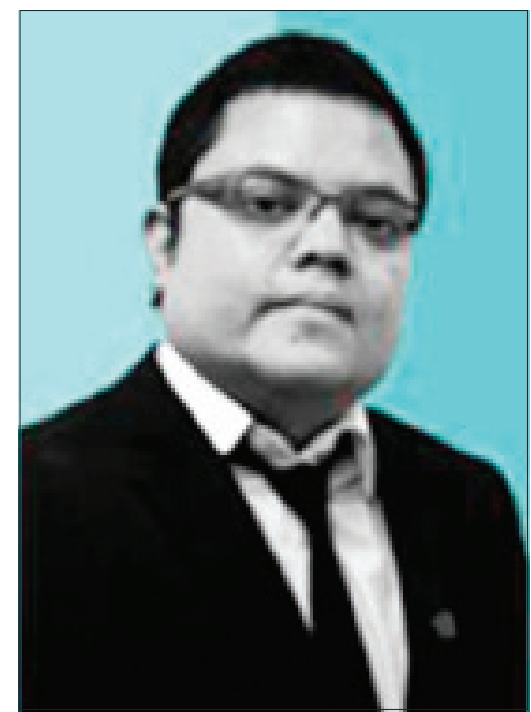

Rodrigo Antonio Colorado

Los antropólogos han definido a la socialidad humana como una serie de posibilidades de coordinación social con otras posibilidades, que están influenciadas por la dinámica de los individuos y grupos sociales (Ibídem).

\subsection{Discurso de Educación inclusiva.}

La presente investigación indagó el proceso de aprendizaje cultural y social de los estudiantes con autismo, en los Centros Educativos de El Salvador, esto debido este entramado social, tiene un programa social y un discurso político de Educación Inclusiva.

Para que podamos hablar de Educación inclusiva es necesario que las Escuelas y Colegios privados nombren sus instituciones como Centro Educativo, este concepto, consiste que la institución es un proyecto común de mejora integral de padres, profesores, alumnos y personal no docente en un contexto específico (Isaacs, 2002, p. 120). El Centro Educativo es un concepto más inclusivo y más apreciativo de la experiencia de la comunidad socialidad y la endoculturación del estudiante con TEA.

Los estudiantes con autismo tienen procesos de aprendizajes diferentes, es por esta razón que el sistema educativo mundial, percibe a este estudiantado a través de sus necesidades, las cuales se le conocen como las Necesidades Educativas Especiales, son las que presenta el educando en el aula, estas deben ser atendidas a través de recursos especiales en la escuela regular (UNICEF, 2001). Los 
estudiantes con autismo forman parte del Discurso Educativo, por esta razón es necesario contextualizar todo tipo de evaluaciones que el estudiante tiene en su proceso de aprendizaje.

Podemos encontrar que la UNICEF ha generado lineamientos sobre cómo se debe de integrar e incluir a la población con Necesidades Educativas Especiales, en este caso los estudiantes con autismo. Para ser más conciso, la educación inclusiva implica que todos los niños y niñas de una determinada comunidad aprendan juntos independientemente de sus condiciones personales, sociales o culturales, incluidos aquellos que presentan una discapacidad (Ibídem, p.44).

\section{Una mirada antropológica del autismo.}

Desde la Antropología, la Arquelogía y la Psiquiatría se está haciendo esfuerzos por entender el Autismo, por ejemplo los investigadores Spikins y Wrigth (2016.), citados por Colorado (2016), consideran que se hace un esfuerzo para comprender el TEA, es una condición social diferente, estas personas están dentro de la sociedad y participan dentro de la cultura material, utilizan la tecnología para comunicarse en las relaciones socioculturales y muchas veces son los conceptos matemáticos la pieza clave para entrar en contacto con nuestro mundo, según las antropólogas Elinor Ochs, Olga Solomon de la Universidad de Los Ángeles (California-EE. UU.), y el antropólogo Roy Grinker de la Universidad George Washington (EE. UU.) padre de una niña con autismo.

También en las Ciencias Sociales, se considera que es necesario comprender las habilidades y destrezas que tienen las personas con autismo, por ejemplo, el antropólogo y psiquiatra Grinker (2010, pp. 172,173) encontró lo siguiente:

Mostrando las formas complejas en las que los niños y adultos con autismo participan y contribuyen a sus sociedades. Al mismo tiempo, los antropólogos han comenzado a contextualizar el debate público sobre la prevalencia del autismo y la etiología de los procesos históricos y culturales.

\section{Diálogo entre Ciencias Sociales y Ciencias de la Salud}

La Antropología, la Arqueología comprenden al ser humano con el TEA, desde sus entornos socioculturales, así como sus habilidades y destrezas y el debate público sobre su inclusión social. Para poder sustentar este visión de las Ciencias Sociales sobre el Autismo, mi sitio web (www.rodrigocolorado.com), tiene el extracto de la entrevista que le realice al Doctor en sociología Damian Milton de la Universidad de Kent-Reino Unido, en esa oportunidad le pregunte i Si las personas con autismo construyen cultura?, el respondió lo siguiente "Creo que las personas con autismo son parte de la cultura, están dentro de ella, y construyen también su propia cultura en sus comunidades y en el mundo social 
que los rodea" (Comunicación personal, Damián, 8 de diciembre de 2016, ¿las personas con autismo construyen cultura?).

Revisando los discursos políticos y educativos que los Gobiernos, las instituciones mundiales educativas, Ciencias Sociales y Ciencias de la Salud, consideran que es necesario analizar y estudiar los entornos socioculturales de los estudiantes con el TEA. Para la antropología es importante estudiar la socialidad y endoculturación del estudiante con el TEA en los Centros Educativos de El Salvador.

\section{Resultados y discusión. Estudio Cuanti-cualitativo}

Tabla 1

Personal capacitado en el Centro Educativo

\begin{tabular}{lccccc}
\hline \multicolumn{2}{c}{ Frecuencia } & Porcentaje & $\begin{array}{c}\text { Porcentaje } \\
\text { válido }\end{array}$ & $\begin{array}{c}\text { Porcentaje } \\
\text { acumulado }\end{array}$ \\
\hline & 1 & 12 & 16,2 & 57,1 & 57,1 \\
& 2 & 4 & 5,4 & 19,0 & 76,2 \\
& 3 & 3 & 4,1 & 14,3 & 90,5 \\
Validos & 4 & 2 & 2,7 & 9,5 & 100,0 \\
& & 21 & 28,4 & 100,0 & \\
Total & & & & 64,9 & \\
Perdidos Sistema & 53 & 71,6 & 35,1 & \\
\multicolumn{2}{c}{ Total } & 74 & 100,0 & 100,0 & \\
& & & &
\end{tabular}

Elaboración propia, 2016.

La pregunta siguiente es: Número de personal capacitado en el Centro Educativo para atender al niño (a), adolescentes y joven con TEA la respuesta por parte de los docentes fue que existe un $57,1 \%$ de los docentes capacitados $(\mathrm{n}=12)$, también un $19,0 \%$ de docentes capacitados $(n=4)$, otro grupo de docentes considera que $14,3 \%(\mathrm{n}=3)$ 4,3 $\%$, mientras que un último resultado de docentes capacitados es de $9.5 \%(n=2)$, un nivel muy bajo de personal capacitado, es decir, que en los Centros Educativos regulares hay docentes muchos docentes sin ninguna capacitación, la atención es bastante empírica, más que tener un apoyo especializado en el tema. 
Tabla 2.

Autismo, metodología e inclusión.

\begin{tabular}{llll}
\hline Hipótesis nula & Test & Sig. & Decisión \\
\hline La distribución En su salón de & Prueba de & Rechazar la \\
clases, ¿Cuántos niños (as), & Mann-Whitney & & Hipótesis nula. \\
adolescentes y jóvenes tienen & de muestras & & \\
TEA es la misma entre categorías & independientes & & \\
de iExiste una metodología & & & \\
en los centros educativos que & & \\
garanticen la integración, la & & \\
inclusión y participación del & & \\
niño (a), adolescente y joven con & & \\
TEA? & & & \\
\hline
\end{tabular}

Se muestra las siguientes asintóticas. El nivel de significancia es, 0.5.

Elaboración propia, 2016.

Como se puede apreciar la hipótesis Nula se contrasta con las preguntas: En su salón de clases, ¿cuántos niños (as), adolescentes y jóvenes que tienen TEA? con ¿Existe una metodología en los centros educativos que garanticen la integración y participación del niño (a), adolescente y joven con TEA? La hipótesis se rechaza con un resultado de significancia de 028 , en donde podemos observar que sí existe relación entre el número de niños con el TEA y la metodología de integración aplicada por los Centros Educativos.

Tabla 3

Metodología

\begin{tabular}{|c|c|c|}
\hline Fuente & Tema & $\begin{array}{l}\text { Abstract } \\
\text { entrevista }\end{array}$ \\
\hline $\begin{array}{l}\text { 30-08-2016 } \\
\text { Entrevista a } \\
\text { profesionales } \quad \text { y } \\
\text { especialista en } \\
\text { trato con niños } \\
\text { (as), adolescentes y } \\
\text { jóvenes con TEA. }\end{array}$ & $\begin{array}{l}\text { ¿Qué tipo de metodología } \\
\text { de la educación lleva a } \\
\text { cabo con los niños y niñas } \\
\text { con TEA? }\end{array}$ & $\begin{array}{l}\text { "Una metodología } \\
\text { integral explorativa. } \\
\text { Adecuada a las } \\
\text { necesidades del grado. } \\
\text { Tomando en cuenta } \\
\text { los conocimientos } \\
\text { previos". }\end{array}$ \\
\hline
\end{tabular}

Elaboración propia, 2016. 
Los docentes tienen una metodología y la adecuan a las circunstancias que se presentan en el aula, en dicha metodología se encuentran los procesos de endoculturación y socialidad de los estudiantes con el TEA, ya que se tiene el diagnóstico médico de ser funcionales, ya que en las evaluaciones de los docentes, los estudiantes mencionados presentan en las evaluaciones un conocimiento previo, por esos los encontramos manejando programas avanzados de la informática, es decir, cuando interviene la institución en el Centro Educativo.

Tabla 4.

Voz suave.

\begin{tabular}{llrrrr}
\hline & Frecuencia & Porcentaje & $\begin{array}{c}\text { Porcentaje } \\
\text { válido }\end{array}$ & $\begin{array}{c}\text { Porcentaje } \\
\text { acumulado }\end{array}$ \\
\hline \multirow{2}{*}{ Válidos } & Nada/No & 7 & 9,5 & 10,6 & 10,6 \\
& Un poco & 2 & 2,7 & 3,0 & 13,6 \\
& Suficiente & 10 & 13,5 & 15,2 & 28,8 \\
& Bastante & & & & \\
& Completamente & 20 & 27,0 & 30,3 & 59,1 \\
Perdidos & Total & 27 & 36,5 & 40,9 & 100,0 \\
& Perdidos & 66 & 89,2 & & \\
& Sistema & 8 & 10,8 & & \\
Total & & & & & \\
& & 74 & 100,0 & & \\
\hline
\end{tabular}

Elaboración propia, 2016.

El análisis descriptivo de la pregunta: ¿Le hablo con una voz suave al niño (a), adolescente y joven con TEA en el aula?, nos dice que existe un 10,6 \% de docentes que respondió nada le habla con voz suave para la inclusión del niño (a), adolescente y joven con TEA $(n=7)$. Otro grupo de docentes dice un poco le habla con voz suave al estudiante con TEA un $3,0 \%$, es decir $(n=2)$. Otro grupo de docentes dice que le habla lo suficiente con vos suave al estudiante con TEA $15,2 \%$, es decir $(n=10)$, mientras que un grupo de docentes considera que le habla bastante con voz suave al estudiante con TEA en el aula es el 30,3\%, siendo $(\mathrm{n}=20)$. Mientras tanto un grupo último de docentes considera que le habla completamente con vos suave al niño con TEA en el aula es de $36,5 \%$, es decir $(\mathrm{n}=27)$, el cual es un resultado positivo, ya que muy a pesar de que no hay una metodología, pero si hay un sentido humano para la atención y la enseñanza 
del estudiante con TEA, los docentes parten de la concientización sobre el tema. A los estudiantes con Autismo es necesario hablarles con una voz suave, por esta razón es importante que en los Centros Educativos se tenga como una de las acciones sistematizadas en la práctica docente, el uso de la voz suave.

Tabla 5

\section{Dios y la intervención en la inclusión educativa}

Fuente

31-08-2016.

Capacitación sobre autismo. ISRI.
Tema

¿Los papás explican a los profesores el Diagnóstico de los niños con TEA?
Abstract de entrevista

"Dios sabe por qué nos ha elegido, bueno en mi familia tenemos a un niño con este problema, créame es bastante duro, Dios sabe por qué ha puesto a cada uno de estos niños en las escuelas y colegios en que estamos trabajando, lo que podemos hacerlo es apoyarlo y amarlos porque son niños, gracias".

Elaboración propia, 2016.

Una de las características fundamentales de la práctica de la educación inclusiva es el trato con dignidad a los estudiantes y este se logra a través de hablarle con voz suave a los educandos, siendo los docentes en su mayoría creyentes cristianos, lo cual garantiza que a través de sus prácticas de creencias religiosas lleven a cabo la adaptación de programas educativos especiales con la faceta de una educación con valores inclusivos.

Comprobación de hipótesis se expresa de la siguiente manera: Se realizó la comprobación de hipótesis con apoyo del programa SPSS en su versión 21, por lo cual se han contrastado variables clasificadas en nominales y de escala. 
Tabla 6

Enseñanza de la inclusión

\begin{tabular}{llll}
\hline Hipótesis nula & Test & Sig. & Decisión \\
\hline Ladistribuciónde ¿Losdocentes & Prueba de U de & & Rechazar la \\
del centro educativo enseñan en & Mann-Whitney & & Hipótesis nula. \\
las clases la inclusión del niño & de muestras & & \\
(a), adolescente y joven con el & independientes & & \\
TEA?, es la misma entre las & & & \\
categorías de ¿Se utilizan póster & & \\
para señalizar las actividades & & \\
a desarrollar en el aula para & & \\
los niños (as), adolescentes y & & \\
jóvenes con el TEA? & & \\
\hline
\end{tabular}

Se muestra las siguientes asintóticas. El nivel de significancia es, 0.5.

Se muestra la significancia exacta para esta prueba.

Elaboración propia, 2016.

Como se observa, el contraste de hipótesis se realizó cruzando las preguntas:

¿Los docentes del Centro Educativo enseñan en las clases la inclusión del niño (a), adolescente y joven con el TEA? con ¿se está utilizando póster para señalizar las actividades a desarrollar en las aulas para los niños (as), adolescentes y jóvenes con el TEA? por medio de una prueba de U de Mann-Whitney de muestras independientes, lo cual dio como resultado la retención de la hipótesis nula, ya que arrojó una significan cía de .00. Esto significa que si existe está relacionado enseñanza de la inclusión con utilización de póster en el aula. Es decir, que los docentes tienen una metodología para la inclusión educativa de los estudiantes con autismo y es el uso de póster para sensibilizar en el tema de respeto e inclusión. 
Tabla 7

El docente e inclusión educativa en el aula

\begin{tabular}{|c|c|c|}
\hline Fuente & Tema & Abstract de entrevista \\
\hline $\begin{array}{l}\text { 15-07-2016. } \\
\text { Entrevista a } \\
\text { profesionales y } \\
\text { especialista en } \\
\text { trato con niños } \\
\text { (as), adolescentes } \\
\text { y jóvenes con } \\
\text { TEA. ISRI. }\end{array}$ & $\begin{array}{l}\text { ¿Considera usted que } \\
\text { el sistema educativo } \\
\text { salvadoreño proporcione } \\
\text { el tratamiento adecuado } \\
\text { para el aprendizaje de los } \\
\text { niños (as), adolescentes y } \\
\text { jóvenes con TEA? }\end{array}$ & $\begin{array}{l}\text { "Es un proceso creo que } \\
\text { el MINED, está entrando } \\
\text { un proceso de inclusión, } \\
\text { el maestro está interesado } \\
\text { con los diagnosticos, } \\
\text { nosotros acá tenemos } \\
\text { capacitaciones, } \\
\text { con los compañeros } \\
\text { encargados de atención } \\
\text { con niños con autismo, } \\
\text { los maestros r están } \\
\text { interesados". }\end{array}$ \\
\hline
\end{tabular}

Elaboración propia, 2016.

Se observa en la tabla 6 en comparación con la Tabla 7 de observación participante, cuando se rechaza la hipótesis correspondiente la enseñanza de la inclusión educativa y el uso de póster, se comprueba con los hallazgos cualitativos encontrados en el taller de capacitación a docentes, que si hay una metodología de la educación inclusiva para la socialidad del estudiante con TEA.

Tabla 8.

Autismo y computadora.

\begin{tabular}{llll}
\hline Hipótesis nula & Test & Sig. & Decisión \\
\hline La distribución de En su & Prueba de U de Mann- & Rechazar la \\
salón de clases, ¿Cuántos & Whitney de muestras & & Hipótesis nula. \\
niños (as), adolescentes y & independientes & .018 & \\
jóvenes tienen el TEA? Es la & & & \\
misma entre las categorías de & & \\
¿En el centro educativo hay & & \\
una computadora asignada & & \\
para que sea utilizada para & & \\
que sea utilizada por los & & \\
niños (as), adolescentes y & & \\
jóvenes con TEA? &
\end{tabular}

Se muestra las siguientes asintóticas. El nivel de significancia es, 0.5.

Elaboración propia, 2016. 
Como se puede apreciar, el contraste de hipótesis se realizó cruzando las preguntas: En su salón de clases ¿Cuántos niños (as), adolescentes y jóvenes tienen el TEA?, con ¿En el centro educativo hay una computadora asignada para que sea utilizada por los niños (as), adolescentes y jóvenes con TEA? por medio de una prueba de U de Mann-Whitney de muestras independientes, lo cual dio como resultado la retención de la hipótesis nula, ya que arrojó una significancia de. 018. Esto significa que sospechas sobre TEA a estudiantes si está relacionada con computadora asignada por los niños (as), adolescentes y jóvenes con TEA.

Se comprueba que no hay computadoras asignadas para el aprendizaje de los estudiantes con autismo en los Centros Educativos.

Tabla 9.

Autismo y computadora.

\begin{tabular}{|c|c|c|}
\hline Fuente & Tema & Abstract de entrevista \\
\hline $19-9-2016$ & niño & "Mire él se sienta adelante, \\
\hline Entrevista & adolescente y joven con & como la clase la recibimos en \\
\hline profesionales & el TEA tiene formas & un centro de cómputo, ya vio \\
\hline especialista & comunicarse & usted que se sienta adelante, \\
\hline $\begin{array}{l}\text { trato con niños } \\
\text { (as), adolescentes y } \\
\text { jóvenes con TEA. }\end{array}$ & $\begin{array}{l}\text { con los familiares y } \\
\text { amigos? }\end{array}$ & $\begin{array}{l}\text { siempre está preguntando, él } \\
\text { se adelanta mucho, el busca en } \\
\text { el navegador". }\end{array}$ \\
\hline $6-9-2016$ & ¿Hago & "Está haciendo actualmente \\
\hline $\begin{array}{l}\text { Entrevista a los niños } \\
\text { (as) compañeros de } \\
\text { aula del estudiante con } \\
\text { TEA. }\end{array}$ & $\begin{array}{l}\text { mi compañerito o } \\
\text { compañerita en el aula } \\
\text { que es muy callado? }\end{array}$ & $\begin{array}{l}\text { una aplicación para Tablet } \\
\text { y celular, y lo estudio en } \\
\text { internet en IBM en el tutorial } \\
\text { desarrolle aplicaciones con } \\
\text { Android con Elipse". }\end{array}$ \\
\hline
\end{tabular}

Elaboración propia, 2016. Ver sitio web (www.rodrigocolorado.com)

Los datos cuantitativos nos presentan un Centro Educativo sin computadoras, siendo esta una herramienta importante para el proceso de endoculturación del estudiante con TEA quien hace buen uso para buscar información e instruirse para lograr sobresalir en la materia de Informática, y en muchos casos construyen herramientas de la informática como las aplicaciones para Tablet, en la mayoría de casos el estudiante con TEA tiene computadora en su casa, lo cual hace el complemento ante la escasez de computadoras en su Centro Educativo. 
Tabla 10.

Autismo con asistencia a biblioteca.

\begin{tabular}{|c|c|c|c|}
\hline Hipótesis nula & Test & Sig. & Decisión \\
\hline $\begin{array}{l}\text { La distribución de En su } \\
\text { salón de clases, ¿Cuántos } \\
\text { niños (as), adolescentes } \\
\text { y jóvenes tiene el TEA? } \\
\text { Es la misma entre las } \\
\text { categorías de ¿Hay una } \\
\text { biblioteca en la escuela } \\
\text { que asiste el niño (a), } \\
\text { adolescente joven con } \\
\text { el TEA? }\end{array}$ & $\begin{array}{l}\text { Prueba de U de } \\
\text { Mann-Whitney } \\
\text { de muestras } \\
\text { independientes }\end{array}$ & .018 & $\begin{array}{l}\text { Rechazar la } \\
\text { Hipótesis nula. }\end{array}$ \\
\hline
\end{tabular}

Se muestra las siguientes asintóticas. El nivel de significancia es, 0.5.

Elaboración propia, 2016.

Como se puede apreciar, el contraste de hipótesis se realizó cruzando las preguntas: En su salón de clases, ¿Cuántos niños (as), adolescentes y jóvenes tienen TEA?, con ¿Hay una biblioteca en el centro educativo que asiste el niño (a), adolescente y joven con TEA? por medio de una prueba de U de MannWhitney de muestras independientes, lo cual dio como resultado la retención de la hipótesis nula, ya que arrojó una significancia de .012. Esto significa que el estudiante con TEA si está relacionada con una biblioteca en el centro educativo que asiste el niño (a), adolescente y joven con el TEA.

Tabla 11.

Uso de libros en el aula.

\begin{tabular}{lll}
\hline $\begin{array}{l}\text { Fuente } \\
\text { (Taller ISRI) }\end{array}$ & Tema & Abstract de capacitación \\
\hline $\begin{array}{l}\text { 29-8-2016. } \\
\text { Diario de Campo }\end{array}$ & $\begin{array}{l}\text { "Luego de hablar cinco minutos } \\
\text { conmigo saca su libro de Lenguaje, } \\
\text { participante }\end{array}$ & $\begin{array}{l}\text { pero es un libro del séptimo grado } \\
\text { del MINED, del año 2007, de }\end{array}$ \\
& $\begin{array}{l}\text { la administración de ministra de } \\
\text { Educación Darlin Meza y del } \\
\text { presidente Antonio Saca". }\end{array}$ \\
\hline
\end{tabular}

Elaboración propia, 2016. 
En el sitio web (www.rodrigocolorado.com) se encuentra la tesis de grado "Transformaciones socioculturales generadas por la convivencia con niños (as), adolescentes y jóvenes con Trastorno de Espectro Autista en los Centros Educativos de los Departamentos de San Salvador, La Libertad, Sonsonate, Ahuachapán, Chalatenango y San Vicente" este documento se presenta el Diagrama 25 (Colorado, p.152), este esquema presenta el análisis cualitativo y cual se realizó con la asistencia herramienta del Software especializado QSR NVIVO 10.

Este análisis permitió codificar, a través de unidades de significado, los conceptos e ideas más comunes las representaciones sociales y culturales de los entrevistados, quienes fueron principalmente profesores, psicólogos y terapistas, La técnica se aplicó mediante una guía de entrevista estructurada semiabierta, la cual permitió a los entrevistados expandirse en sus respuestas y profundizar de acuerdo con las ideas y nociones que se tenían respecto al tema de investigación.

A partir de la identificación de dichas unidades de análisis, se procedió a hacer un análisis de clúster, el cual nos permitió hacer una relación por conglomerados de las vinculaciones que existen entre los diferentes significados de acuerdo con el asocio que hacen los actores sociales que interactúan en el entorno del estudiante con TEA, en este sentido, son los niños (Colorado, p.152), los que más asimilan la cultura de Educación Inclusiva. Esta relación se traza mediante un procedimiento de modelado de correlación de ideas.

\section{Figura 1, Resultado de asociación de Nodos.}

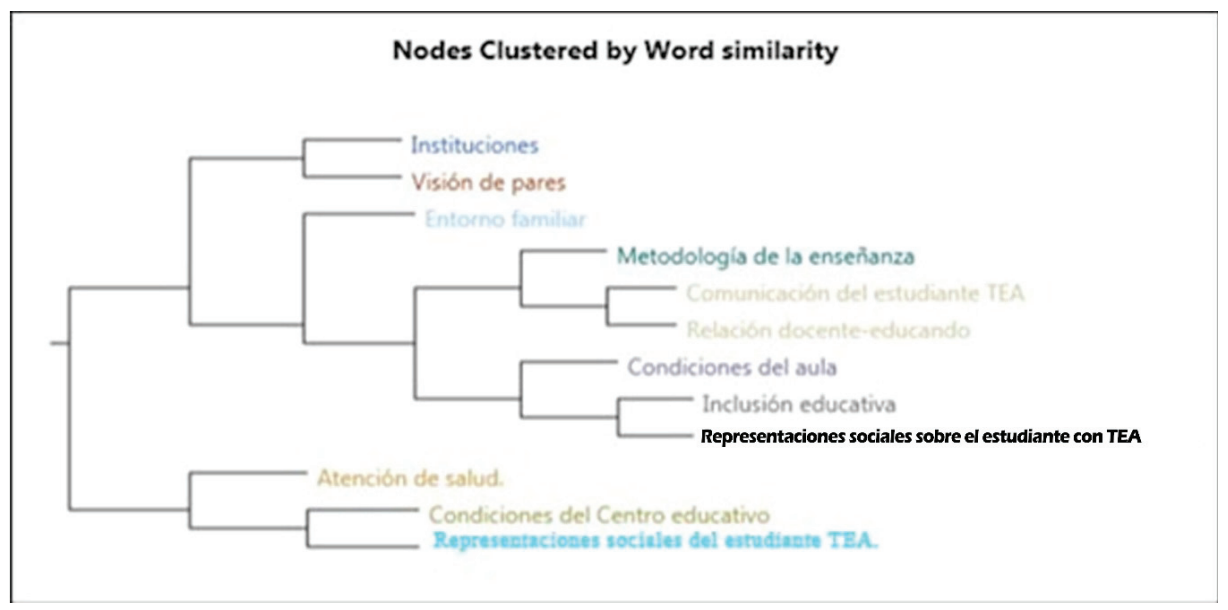

Ver sitio web (www.rodrigocolorado.com) (Ibídem, p.156). 
Los nodos Representaciones sociales, estudiante con el TEA, con condiciones del Centro educativo y, están relacionadas con la atención de salud. Podemos decir que dentro del proceso de endoculturación y socialidad del estudiante TEA, existen las condiciones que son apropiadas para que el estudiante tenga una experiencia agradable en el proceso de aprendizaje, es decir, que se debe a la capacidad de gestión que las Instituciones tienen en los Centros Educativos. Por ejemplo, en el siguiente ejemplo que se presenta en la Tabla 12, se observa la influencia que tiene el padre con el especialista de la salud y de cómo este incide en la buena salud de su hijo en el Centro Educativo.

Tabla 12.

Padre de familia y salud.

\begin{tabular}{|l|l|l|}
\hline $\begin{array}{l}\text { Fuente } \\
\text { (Taller ISRI) }\end{array}$ & Tema & Abstract de capacitación \\
\hline $\begin{array}{l}\text { Entrevistas a padres } \\
\text { de Familia }\end{array}$ & $\begin{array}{c}\text { ¿Ha tenido } \\
\text { un impacto en } \\
\text { la conducta la } \\
\text { atención del } \\
\text { psicólogo con su } \\
\text { hijo? }\end{array}$ & $\begin{array}{l}\text { "Cuando lo lleve al psicólogo } \\
\text { ella decía que él estaba } \\
\text { estresado, estuvo todo un año } \\
\text { en terapia académica todo un } \\
\text { año, para que no perdiera el } \\
\text { año, la gente de Music Time } \\
\text { ha visitado el Colegio para } \\
\text { conocer como es caso } 6, \\
\text { este es el método que 6 le ha } \\
\text { funcionado". }\end{array}$ \\
& & \\
&
\end{tabular}

Elaboración propia, 2016.

La intervención del padre que tiene hijo con el TEA es importante ya que garantiza la salud del educando y por tanto garantiza también la intervención de la institución en el centro educativo en este caso Music Time, esto garantiza que los docentes y pares se informen de la situación que el estudiante con autismo tiene en el proceso de endoculturación y socialidad, las cuales se ven garantizadas en las Representaciones Socioculturales tales como la tolerancia, el respeto a la diversidad, en si una cultura de educación inclusiva.

Los nodos Representaciones sociales sobre el estudiante con el TEA e inclusión educativa están relacionados a las condiciones del Aula, podemos inferir que, a mejores condiciones del aula, mejor será la educación inclusiva, es decir, el estudiante con el TEA será sobresaliente, si el entorno educativo garantiza las posibilidades para desarrollarse como estudiante. 


\section{Conclusión}

El estudio cuantitativo nos da la calidad de la muestra a través de la recolección de dato expresado en una encuesta que se les paso a 74 docentes, el sexo de los encuestados es masculino con 7 docentes y femenino con un 67, la mayoría mujeres de 25 años, provenientes de 37 escuelas públicas y 9 colegios privados, los Centros Educativos encuestados, tienen 65 casos de estudiantes con autismo, en los 6 departamentos: San Salvador, La Libertad, Chalatenango, Sonsonate, San Vicente, Ahuachapán, ver sitio web (www.rodrigocolorado.com). Dentro de estos Centros Educativos la mayoría de docentes no están capacitados para afrontar el tema de los estudiantes con Necesidades Especiales Educativas, no hay un personal especializado apoyando dichos Centros Educativos, pero a pesar de esta situación, los docentes y los pares han sabido afrontar esta realidad, a través de promoción de valores positivos que representan la Educación inclusiva, esto se da más en la educación inicial, ya que en esta etapa es donde no se refleja las diferencias entre un estudiante con Autismo y un niño (a) "normal", los colegios privados con atención especializada están iniciando un proceso educativo, pero es una experiencia que no está sistematizada con las instituciones y asociaciones que trabajan el tema del autismo. Se aclara que esta investigación es de febrero a diciembre de 2016.

Con referente a las condiciones en que se encuentran las aulas de los Centros Educativos encuestados, en el caso de las Escuelas públicas y algunos Colegios privados, pues su estado es crítico, no son aulas adecuadas para que un estudiante con autismo pueda recibir sus clases. Una de los matices del discurso de la Educación Inclusiva en el ambiente de convivencia es la utilización de póster o material didáctico en las aulas de los Centros Educativos, ya que garantizan el proceso de educación, endoculturación, adaptación, la socialidad del estudiante que padece el TEA, lo cual, si se da, con los valores como el respeto a la diversidad de opinión y a la diversidad cultural, sin embargo, pero no está explicito el tema del respeto a los estudiantes con Necesidades Educativas Especiales.

Con referente a la utilidad de la computadora con el aprendizaje del estudiante es necesaria y se hace posible su proceso de aprendizaje más rápido y más significativo, ya que se especializa en el tema. Como podemos observar es necesario que los Centros Educativos y especialmente las Escuelas Públicas que llevan a cabo el programa de Escuela inclusiva, tienen que impartirles capacitaciones a los docentes para que puedan enfrentarse a los problemas que el estudiante con autismo se enfrenta, por ejemplo: ¿Cómo se va a sociabilizar con su entorno educativo?, ¿es posible la socialidad del estudiante con autismo con sus pares y docentes? 
La capacitación del docente garantiza que los espacios culturales y educativos se vayan abriendo camino para el estudiante con autismo, ya que el Centro Educativo forma valores y como hemos podido apreciar es que es la endoculturación la que ha permitido tener una representación social positiva del estudiante con autismo, también el estudiante con autismo ha tenido una buena representación social de su entorno, dándose así una dicotomía entre el entorno social, la intersubjetividad y la pertenencia de grupo, ya que según el estigma o el tabú sobre el autismo, la persona no se puede socializar en su entorno educativo.

Si la capacitación del docente girara entorno a la inclusión educativa de los entornos sociales y culturales comunitarios girarían entorno al Centro Educativo, pues si habría realmente incidencia en los cambios de una cultura verticalista y autoritaria que no acepta a las personas con autismo en los Centros Educativos es decir, se puede establecer un trabajo cultural y educativo a partir de la premisa de inclusión educativa, ya que en la actualidad no se ha logrado crear una plataforma en común entre el Centro Educativo, asociaciones de padres con hijos con autismo, MINED e Instituciones que trabajan el tema.

En este estudio cualitativo se apreció que las experiencias de cada padre, docente y profesional de la salud que tienen de los estudiantes con el TEA, están constituidas exclusivamente por las relaciones que el padre tiene con los que lo rodean, las peculiaridades de las experiencias que los padres tienen con sus hijos en los entornos familiares, de salud y el del centro educativo.

De esta manera, la investigación consiste en describir la experiencia individual de: algunos niños (as), adolescentes, padres y profesionales de la salud, que luchan por la inclusión educativa en los Centros Educativos de El Salvador, casi todos luchan contra las representaciones sociales como el acoso escolar que genera la ansiedad en los estudiantes con TEA.

La socialidad de los estudiantes con el TEA en los Centros Educativos, se expresa así: La observación participante y la entrevista han garantizado conocer los 9 casos de los estudiantes niños (as), adolescentes y jóvenes con TEA, por lo cual en el ámbito de las habilidades sociales y los impedimentos muestran una mayor propensión a la socialidad de lo comúnmente asumido. Las posibilidades de la coordinación social están organizadas en parte por la comunicación intersubjetiva y el proceso de endoculturación de las familias, los terapeutas, los maestros y otros interlocutores con los que los niños (as), adolescente y jóvenes con autismo interactúan diariamente.

Los padres que asisten a las charlas de capacitación en el ISRI, ASA, Asociación angelitos de Arcatao y Music Time, dan a conocer su experiencia y enseñan a otros padres su experiencia en la educación e inclusión social de estudiantes con 
TEA, misma situación sucede con los docentes que asisten a las capacitaciones del MINED.

La Antropología nos garantiza hacer un análisis reflexivo sobre el Autismo en los Centros Educativos de El Salvador, ya que las personas con autismo se consideran como un sujeto en el algoritmo social de la comunidad humana. Las personas con autismo necesitan comunidades adecuadas para prosperar a través de sus talentos, en muchos casos las comunidades necesitan individuos con autismo (Spikins y Wrigth, 2016). En el plano educativo podemos traducirlo que es necesario establecer un puente de comunicación que fortalezca la Educación Inclusiva, esta debe tomar encuenta la participación de los padres de familia, docentes, especialistas de la salud, asociaciones que trabajan el tema autismo, construyendo así un Centro Educativo de convivencia. En donde todos los involucrados en una comunidad comparten un nivel de compromiso mutuo, actividad o interés común a través del cual aprenden prácticas comunes (Milton, 2015), en este caso son los Centros Educativos de El Salvador, que realizan prácticas educativas-culturales inclusivas a través de los valores cristianos-humanistas que los docentes enseñan y practican en sus aulas, en el caso de la sensibilización en los Centros Educativos y los programas: Sub-Programa Vaso de Leche (Ibídem) y Programa de Alimentación y Salud Escolar (Colorado, p.125)

\section{Referentes Bibliográficos.}

Colorado, R. (2016). "Transformaciones socioculturales generadas por la convivencia con niños (as), adolescentes y jóvenes con Trastorno de Espectro Autista en los Centros Educativos de los Departamentos de San Salvador, La Libertad, Sonsonate, Ahuachapán, Chalatenango y San Vicente". Recuperado de https: www.rodrigocolorado.com

Colorado, R. (2017). El autismo, una pieza para el rompecabezas de la evolución humana (Artículo de divulgación).

Recuperado de http://autismodiario.org/2017/06/13/autismo-una-piezarompecabezas-la-evolucion-humana/

Eumed. (2012). Enfoques cualitativo, cuantitativo y mixto. Recuperado de http:// www.eumed.net/tesis- doctorales/2012/ mirm/cualitativo_cuantitativo mixto.html.

Geta, P. (2011). Antropología: Contribución al estudio de la educación. Revista portuguesa de pedagogía. Recuperado de http://digitalisdsp. uc.pt/bitstream/ 10316.2/ 5301/2/9\%20-\%20Antropologia \%20 Contribucion $\% 20$ al $\% 20$ Estudio $\%$ 20de $\% 201$ a $\% 20$ Educacion.pdf? $\ln =$ es. 
Grinker, R. (2010). Commentary: On Being Autistic, and Social. 172-177 doi:10.1111/j.1548-1352.2010. 01087.x

Harris, M. (2004). Antropología cultural. Madrid: Alianza editorial.

Isaacs, D. (2002). Centro educativo ¿Organización o Comunidad? Revista estudios sobre educación. Recuperado de http://dadun.unav.edu/bitstream /10171/8035/1/Estudios\%206.pdf.

Medline Plus. (2016). Trastorno del espectro autista. Recuperado de https:// medlineplus.gov/spanish/autismspectrumdisorder.html.

Milton, D. (2015). Educational discourse and the autistic student: a study using Q-sort methodology. (Tesis doctoral). University of Birmingham, Birmingham, GB. Recuperado de http://etheses.bham.ac.uk/6505/1/ Milton16PhD_Redacted.pdf

MINED. (2015). Programas sociales. Recuperado de http://www.mined.gob. sv/index.php/programas-sociales

MINED. (2018). Una Niña, Un Niño, Una Computadora. Recuperado de http:// www.mined.gob.sv/index.php/noticias/item/9215-mas-computadoraspara-el-programa-presidencial-una-nina-un-nino-una-computadora

Mora, M. (2002). Las teorías de las representaciones sociales de Sergei Moscovici. Revista Athenea Digital. Recuperado de http://www.raco.cat/ index.php /Athenea/article/viewFile/34106/33945

Ochs, E. \& Solomon, O. (2010). Autistic Sociality. ETHOS. 1, 12. doi: 10.1111/ j.1548-1352.2009.01082. x.

Spikins, P., \& Wright, B. (2016). The Prehistory of Autism. Recuperado de https:// roundedglobe.com/html/9673edbf-0ba5-47b1-97bd-16ef244fd148/ en/ The $\% 20$ Prehistory $\% 20$ of $\% 20$ Autism/\#c4.

Rojas, R. (2003). Guía para realizar investigaciones sociales. México, D.F.: Plaza y Valdes editores.

UNICEF. (2001). Ciclo de debates: desafíos de la política educacional. Inclusión de niños con discapacidad en la escuela regular. Recuperado de http:// www.unicef.cl/archivos_documento/47/debate8.pdf. 


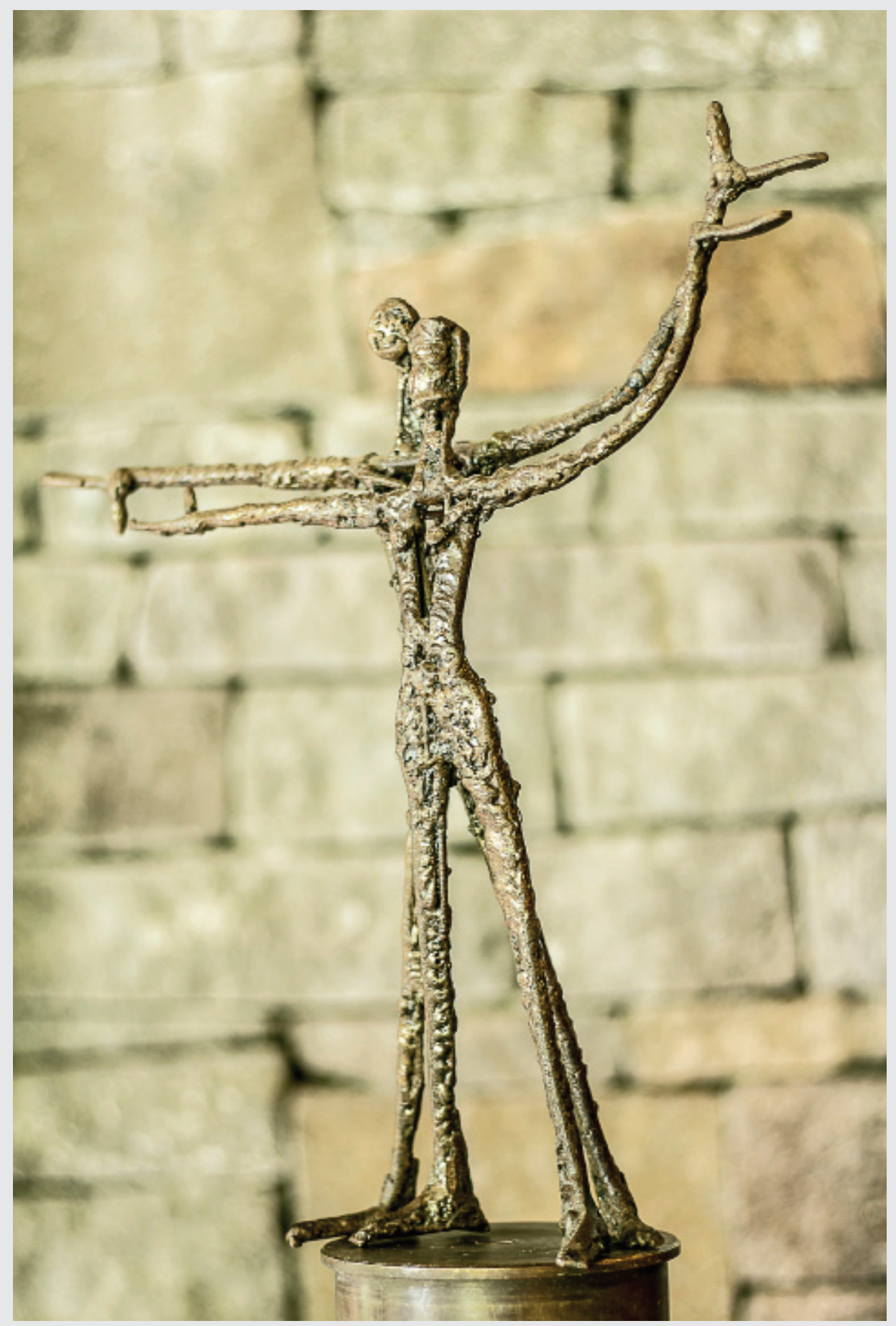

Rubén Martínez Bulnes 